\title{
Linear stability and instability of ion-acoustic plasma solitary waves
}

\author{
Mariana Haragus ${ }^{\mathrm{a}}$, Arnd Scheel ${ }^{\mathrm{b}, *}$ \\ a Mathématiques Appliquées de Bordeaux, Université Bordeaux 1, 351, Cours de la Libération, 33405 Talence Cedex, France \\ ${ }^{\mathrm{b}}$ Institut für Mathematik I, Freie Universität Berlin, Arnimallee 2-6, 14195 Berlin, Germany \\ Received 9 November 2001; accepted 19 April 2002 \\ Communicated by I. Gabitov
}

Dedicated to Klaus Kirchgässner on the occasion of his 70th birthday

\begin{abstract}
The Euler-Poisson equations for a cold, collisionless plasma support ion-acoustic solitary waves. We prove that these waves are spectrally stable at low amplitude in one space-dimension and present numerical evidence that they destabilize at finite amplitude before they develop singularities.
\end{abstract}

(C) 2002 Elsevier Science B.V. All rights reserved.

PACS: $52.35 . \mathrm{Mw} ; 52.35 . \mathrm{Sb}$

Keywords: Ion-acoustic plasma; Solitary waves; Stability

\section{Introduction}

We investigate one-dimensional ion-acoustic solitary waves in a collisionless plasma, when the electron temperature $T_{\mathrm{e}}$ is much higher then the ion temperature $T_{\mathrm{i}}$. Neglecting thermal pressure, we describe the ions by the hydrodynamic equations only:

$$
n_{t}+(n v)_{x}=0, \quad v_{t}+\left(\frac{1}{2} v^{2}+\frac{e}{M} \varphi\right)_{x}=0,
$$

where $n$ denotes the density of the ions, $v$ the velocity, $\varphi$ the electric potential, $M$ the mass of ion, and $e$ the elementary charge. We assume the electrons are distributed according to the Boltzmann law $n_{\mathrm{e}}=n_{0} \mathrm{e}^{e \varphi / T_{\mathrm{e}}}$, and close the system by the Poisson equations for the electric potential $\varphi$ :

$$
\varphi_{x x}+4 \pi e\left(n-n_{0} \mathrm{e}^{e \varphi / T_{\mathrm{e}}}\right)=0 .
$$

\footnotetext{
* Corresponding author. Permanent address: School of Mathematics, University of Minnesota, 206 Church Street S.E., Minneapolis, MN 55455, USA.

E-mail address: scheel@math.umn.edu (A. Scheel).
} 
After an appropriate rescaling, we find the usual Euler-Poisson equations:

$$
n_{t}+(n v)_{x}=0, \quad v_{t}+\left(\frac{1}{2} v^{2}+\varphi\right)_{x}=0, \quad \varphi_{x x}-\mathrm{e}^{\varphi}+n=0 .
$$

These equations are Galilean invariant and reversible, i.e. $(n, v+c, \varphi)(x-c t, t)$ and, respectively, $(n, v, \varphi)(-x,-t)$ are solutions, if $(n, v, \varphi)(x, t)$ is a solution. We refer to [15,21,29] and the references therein for more information on the physical background of these equations.

A particular solution is found for constant density $n \equiv 1$, vanishing potential $\varphi \equiv 0$, and constant velocity $v \equiv v_{0}$; here $v_{0}$ is arbitrary due to Galilean invariance. Linearizing about this spatially homogeneous state we find the linear problem

$$
n_{t}+v_{0} n_{x}+v_{x}=0, \quad v_{t}+v_{0} v_{x}+\varphi_{x}=0, \quad \varphi_{x x}-\varphi+n=0
$$

with sinusoidal travelling-wave solutions $\mathrm{e}^{\mathrm{i}(\omega t-k x)}$, where $\omega$ and $k$ are related through the dispersion relation:

$$
\left(\omega-v_{0} k\right)^{2}\left(k^{2}+1\right)-k^{2}=0 .
$$

Solving for the frequency $\omega$, we find

$$
\omega_{ \pm}(k)=k\left(v_{0} \pm \frac{1}{\sqrt{k^{2}+1}}\right) .
$$

In particular, for supersonic speed $v_{0}>1$, group velocities $\omega_{ \pm}^{\prime}(k)$ are strictly positive. At sonic speed, $\omega_{-}^{\prime}(0)=0$ and for small wave numbers, we can expand

$$
\omega_{-}(k)=\frac{1}{2} k^{3}+\mathrm{O}\left(k^{5}\right)
$$

The cubic term together with Galilean invariance suggests that, in the long-wavelength limit $k \sim 0$ and $n, v \sim 1$, $\varphi \sim 0$, a Korteweg-de Vries equation would govern the dynamics. A natural question then is how far the dynamics of the Korteweg-de Vries equation can actually be found in this particular model for an ion-acoustic plasma. Up to now, only formal derivations of the Korteweg-de Vries limit seem to be known [12,32]; we refer to [16,9,30,4] for validation of the long-wavelength limit in slightly different contexts.

One of the most striking phenomena in the Korteweg-de Vries equation—which largely motivated its discoveryare solitary waves. In particular, solitary waves are among the most simple phenomena which necessitate a truly nonlinear analysis, going beyond the linear dispersion relation (1.4). Existence of solitary waves has been proved in many other physical systems exhibiting a similar cubic expansion of the dispersion relation at the origin. Methods include calculus of variations [8], abstract fixed point arguments and implicit function theorem [3,11], and spatial dynamics $[19,20]$. However, proofs of the stability of solitary waves rely almost exclusively on variational methods, exploiting definiteness of the energy restricted to a fixed value of the impulse functional $[5,6]$. There does not seem to be a single complete proof of stability for a solitary wave which does not satisfy the above criterion.

Opposed to these sufficient stability criteria, spectral investigations provide necessary conditions for stability. Given a solitary wave solution, one investigates the linearization with spectral methods. Absence of spectrum in the right complex half plane is necessary - though far from sufficient - for stability. The work of Pego and Weinstein $[25,26]$ provides an example where the linear, spectral information could be exploited to show asymptotic stability of solitary waves, improving on orbital stability as concluded from energy methods.

A central tool for the study of spectral properties is the Evans function, first introduced in [10]. It allows for a detection of eigenvalues inside and beyond the essential spectrum. Roughly speaking, the Evans function is a Wronskian-like complex function depending on the spectral parameter $\lambda$, whose zeroes coincide with the eigenvalues of the linearized operator. Analyticity allows for counting eigenvalues in the point spectrum with their multiplicity 
in a robust way. Analytic extension of the Evans function across the essential spectrum then gives control on possible unstable eigenvalues created by perturbations.

The plasma equations investigated in the present article, are easily shown to possess a family of solitary waves, asymptotic to $n=1$ and $v=\varphi=0$, parameterized by the wave speed $c>0$ (see also Section 2). Our objective here is to show that the linearization about these solitary waves does not possess any unstable eigenvalues, at least for sufficiently small amplitudes of the solitary wave. As a consequence, we show that the linearized system is asymptotically stable in exponentially weighted spaces.

The Korteweg-de Vries equation as a universal model equation arises in many other physical systems in the long-wavelength limit. The original example are free surface water-waves, where solitary waves have been found for Froude number close to 1, both with strong and without surface tension. Pego and Weinstein [27] proved spectral stability of solitary waves for several Boussinesq model equations arising in this context. It does not come as a surprise that their analysis in the small wavenumber regime is indeed very close to the analysis here (see Section 6.2).

However, a major difference between the plasma system and model equations like the Korteweg-de Vries or Boussinesq equations is that, for high wavenumbers $k$, the system is not dispersive, but the dynamics is to leading order governed by a system of quasilinear conservation laws. This introduces difficulties in the study of the eigenvalue problem at high frequencies (see Section 5).

The same problems arise in the full water-wave problem, both for zero and large surface tension. The methods developed in [14] show that in both cases spectral stability holds in bounded regions of the complex plane, with a subtlety in the case of large surface tension, Weber number $b>1 / 3$. The present work is to our knowledge the first proof of linear stability for a quasilinear system of Hamiltonian equations exhibiting solitary waves.

Solitary waves exist for speed $1<c<c_{\max } \sim 1.5852$. At $c=c_{\max }$, the second derivative blows up at the maximum of the solitary wave. Stability at finite amplitude with respect to one-dimensional perturbations is central for understanding of the plasma turbulence, but is not known. Computing numerically the dependence of the impulse on the wave speed, we derive numerical evidence that the solitary waves are unstable for $c_{\text {crit }}<c<c_{\max }$, where $c_{\text {crit }} \sim 1.52603$.

The paper is organized as follows. We briefly review the existence proof in Section 2 and present our main results in Section 3. The main tools used in the spectral analysis are introduced in Section 4. The stability proof then proceeds in two steps, excluding unstable eigenvalues with high and finite but non-zero-frequency first (Section 5) and with small frequency (Section 6) then. In Section 7, we review the Hamiltonian formulation and the criterion on instability, based on the second derivative of the Evans function and the impulse. We then present numerical results on the instability for $c>c_{\text {crit }}$.

\section{Existence of solitary waves}

We review some properties of the solitary waves and refer to [22] for a detailed proof of existence.

We look for travelling-wave solutions to $(1.1)$ of the form $(1+\tilde{n}, v, \varphi)(x-c t)$ with speed $c$, which decay to zero as $\xi=x-c t \rightarrow \pm \infty$. Such solutions satisfy the system (we write again $x$ for $\xi$ ):

$$
-c \tilde{n}_{x}+((1+\tilde{n}) v)_{x}=0, \quad-c v_{x}+\left(\frac{1}{2} v^{2}+\varphi\right)_{x}=0, \quad \varphi_{x x}-\mathrm{e}^{\varphi}+1+\tilde{n}=0 .
$$

We integrate the first two equations and set the constant of integration to zero, solve for $\tilde{n}$ and $v$ as functions of $\varphi$, and arrive at

$$
\tilde{n}=\frac{c-\sqrt{c^{2}-2 \varphi}}{\sqrt{c^{2}-2 \varphi}}, \quad v=c-\sqrt{c^{2}-2 \varphi}, \quad \varphi_{x x}=\mathrm{e}^{\varphi}-\frac{c}{\sqrt{c^{2}-2 \varphi}} .
$$



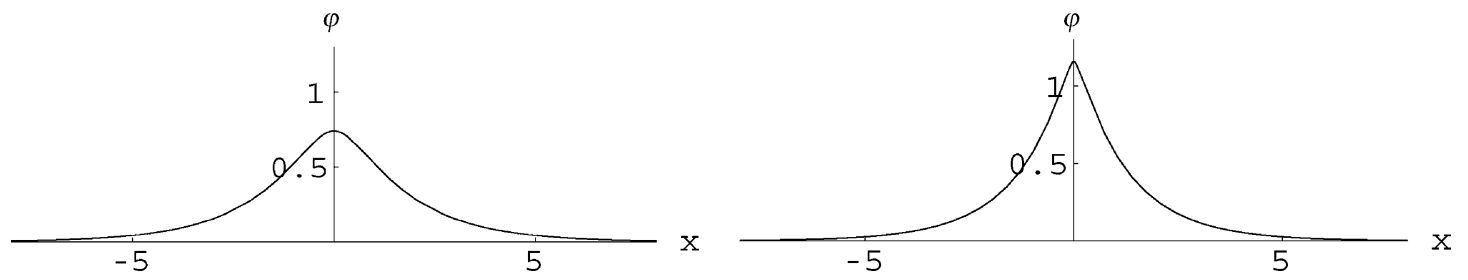

Fig. 1. The shape of the $\varphi$-component of the solitary wave for $c=1.3$ and $c=c_{\max }$.

Solitary waves exist for supersonic speeds $c>1$. For slightly supersonic speed $c=\sqrt{1+\mu}, \mu>0$, we can expand

$$
\varphi_{x x}=\mu \varphi-\varphi^{2}+\mathrm{O}\left(|\varphi|\left(|\mu|^{2}+|\varphi|^{2}\right)\right) .
$$

A solitary wave is a homoclinic solution to the origin of this equation which we develop as

$$
\varphi_{*}(x ; \mu)=\mu \Phi_{*}(\sqrt{\mu} x)+\mu^{2} R(\sqrt{\mu} x)
$$

with $R(y) \leq C \exp (-|y| / 2)$, uniformly in $y \in \mathbb{R}$ and $\mu>0$ small. The leading order term $\Phi_{*}$ solves

$$
\Phi_{*}^{\prime \prime}=\Phi_{*}-\Phi_{*}^{2}
$$

and is explicitly given as $\Phi_{*}(y)=3 / 2 \operatorname{sech}^{2}(y / 2)$. For the full system, the solution is readily expanded as

$$
\left(n_{*}, v_{*}, \varphi_{*}\right)(x)=\mu \Phi_{*}(\sqrt{\mu} x)(1,1,1)+\mu^{2} \tilde{R}(\sqrt{\mu} x)
$$

and again $\tilde{R}(y) \leq C \exp (-|y| / 2)$, uniformly in $y \in \mathbb{R}$ and $\mu>0$ small.

This solution exists for values of the wave speed $c$, until the maximum of the solitary wave reaches the branch point of the square root $\sqrt{c^{2}-2 \varphi}$. This occurs at $c_{\max } \sim 1.5852$, the solution of the equation $\exp \left(c^{2} / 2\right)=1+c^{2}$. Close to the maximum, at $x=0$, the solitary wave for $c=c_{\max }$ scales like

$$
\varphi(x) \sim \frac{1}{2} c_{\max }^{2}-|x|^{4 / 3}\left(\frac{81}{32}\right)^{1 / 3} c_{\max }^{2 / 3} .
$$

We included plots of $\varphi(x)$ for $c=1.3$ and $c=c_{\max }$ (see Fig. 1).

Note also that the solution $\left(n_{*}, v_{*}, \varphi_{*}\right)(x)$ above is an even function, and that we have in fact a one-parameter family of solitary waves, for each speed $c$, due to the invariance of the system under spatial translations.

\section{Spectral and linear stability—main results}

In this section we formulate the linearized problem and state the main results on spectral and linear stability.

Linearizing the Euler-Poisson equations (1.1) about the solitary wave solution (2.3) in a frame moving with the speed $c=\sqrt{1+\mu}>1$ of the solitary wave, we find the system

$$
\begin{aligned}
& n_{t}-c n_{x}+v_{x}+\left(n_{*}(x) v\right)_{x}+\left(v_{*}(x) n\right)_{x}=0, \quad v_{t}-c v_{x}+\varphi_{x}+\left(v_{*}(x) v\right)_{x}=0, \\
& \varphi_{x x}+n-\mathrm{e}^{\varphi_{*}(x)} \varphi=0 .
\end{aligned}
$$

Since $\partial_{x x}-\mathrm{e}^{\varphi_{*}(x)}$ is a small, bounded perturbation of $\partial_{x x}-\mathrm{id}$, we can solve the last equation for $\varphi=\varphi[n]$. Substituting the result into the second equation, we find

$$
\frac{\mathrm{d}}{\mathrm{dt}}\left(\begin{array}{c}
n \\
v
\end{array}\right)=\mathcal{L}_{*}(\mu)\left(\begin{array}{l}
n \\
v
\end{array}\right)
$$


with

$$
\mathcal{L}_{*}(\mu)\left(\begin{array}{l}
n \\
v
\end{array}\right):=\left(\begin{array}{c}
c n_{x}-v_{x}-\left(n_{*}(x) v\right)_{x}-\left(v_{*}(x) n\right)_{x} \\
c v_{x}-\varphi[n]_{x}-\left(v_{*}(x) v\right)_{x}
\end{array}\right) .
$$

We consider $\mathcal{L}_{*}(\mu)$ as a closed operator on $X=L^{2}(\mathbb{R}) \times H^{1}(\mathbb{R})$, with domain of definition $X^{1}=H^{1}(\mathbb{R}) \times H^{2}(\mathbb{R})$.

We say that $\lambda \in \mathbb{C}$ is in the essential spectrum $\operatorname{spec}_{\text {ess }} \mathcal{L}_{*}(\mu)$, if the operator $\lambda \mathrm{id}-\mathcal{L}_{*}(\mu)$ is not Fredholm with index zero. We say that $\lambda \notin \operatorname{spec}_{\text {ess }} \mathcal{L}_{*}(\mu)$ is in the point $\operatorname{spectrum} \operatorname{spec}_{\mathrm{pt}} \mathcal{L}_{*}(\mu)$, if $\lambda$ id $-\mathcal{L}_{*}(\mu)$ is not invertible.

The main result in this paper is the following theorem on the spectrum of $\mathcal{L}_{*}(\mu)$, which shows that the solitary waves are spectrally stable at small amplitudes.

Theorem 1 (Spectral $L^{2}$-stability). There is $\mu_{0}>0$ such that for all $0<\mu<\mu_{0}$, the spectra of $\mathcal{L}_{*}(\mu)$ in $X=L^{2}(\mathbb{R}) \times H^{1}(\mathbb{R})$ satisfy

$$
\operatorname{spec}_{\text {ess }} \mathcal{L}_{*}(\mu)=\mathrm{i} \mathbb{R}, \quad \operatorname{spec}_{\mathrm{pt}} \mathcal{L}_{*}(\mu)=\emptyset .
$$

We consider $\mathcal{L}_{*}(\mu)$ on exponentially weighted spaces next. Define

$$
L_{\eta}^{2}(\mathbb{R})=\left\{u \in L_{\mathrm{loc}}^{2}(\mathbb{R}) ;|u|_{L_{\eta}^{2}}<\infty\right\}
$$

with

$$
|u|_{L_{\eta}^{2}}^{2}=\int_{\mathbb{R}}\left|u(x) \mathrm{e}^{\eta x}\right|^{2} \mathrm{~d} x .
$$

Analogously, we define

$$
H_{\eta}^{1}(\mathbb{R})=\left\{u \in H_{\mathrm{loc}}^{1}(\mathbb{R}) ;|u|_{H_{\eta}^{1}}:=\left|u_{x}\right|_{L_{\eta}^{2}}+|u|_{L_{\eta}^{2}}<\infty\right\} .
$$

We note that similar weighted spaces have been used by Pego and Weinstein [26] in their proof of asymptotic stability of solitary waves for the Korteweg-de Vries equation.

Theorem 2 (Spectral stability in exponential weights). There are positive constants $\mu_{0}, \eta_{0}^{ \pm}$, such that for all $0<\mu<\mu_{0}$ and $0<\eta<\eta_{0}^{+} \mu^{1 / 2}$, there exists $\delta(\eta, \mu)>0$, such that the spectra in the exponentially weighted spaces $X_{\eta}=L_{\eta}^{2}(\mathbb{R}) \times H_{\eta}^{1}(\mathbb{R})$ satisfy

$$
\operatorname{spec}_{\mathrm{ess}}^{\eta} \mathcal{L}_{*}(\mu) \subset\{\lambda ; \operatorname{Re} \lambda \leq-\delta(\eta, \mu)<0\}
$$

and

$$
\operatorname{spec}_{\mathrm{pt}}^{\eta} \mathcal{L}_{*}(\mu) \cap\{\lambda ; \operatorname{Re} \lambda>-\delta(\eta, \mu)\}=\{0\} .
$$

The eigenvalue 0 is of algebraic multiplicity 2, with eigenfunction given by the derivative of the solitary wave $\left(n_{*}, v_{*}\right)$ with respect to $x$. A principal eigenvector is provided by the derivative of $\left(n_{*}, v_{*}\right)$ with respect to the wave speed $c$.

Moreover, for $\eta_{0}^{-} \mu<\eta<\eta_{0}^{+} \mu^{1 / 2}$, we have uniform estimates of the resolvent in $\operatorname{Re} \lambda>-\delta(\eta, \mu)$ with a $1 / \lambda^{2}$-pole at the origin, and

$$
\left\|\left(\lambda \mathrm{id}-\mathcal{L}_{*}(\mu)\right)^{-1}\right\|_{X_{\eta} \rightarrow X_{\eta}} \leq \frac{M}{\operatorname{Re} \lambda+\eta}
$$

uniformly in $\operatorname{Re} \lambda>-\delta(\eta, \mu)$ and outside a neighborhood of the origin. 
The proof of Theorem 2 occupies Sections 5 and 6. In the next section, we introduce the basic tools for the analysis, prove the claims on essential spectra, and show how Theorem 1 is deduced from Theorem 2.

An immediate consequence of Theorem 2 is linear asymptotic stability of the family of solitary waves in weighted spaces. Since the double zero eigenvalue, generated by the tangent space of the two-parameter family of solitary waves, belongs to the point spectrum, we can define a spectral projection $P(\mu, \eta)$ onto the generalized eigenspace for any $0<\mu<\mu_{0}$ and $0<\eta<\eta_{0}^{+} \mu^{1 / 2}$.

Corollary 3.1 (Linear convective stability). There exist positive constants $\mu_{0}, \eta_{0}^{ \pm}$, such that for all $0<\mu<\mu_{0}$ and $\eta_{0}^{-} \mu<\eta<\eta_{0}^{+} \mu^{1 / 2}$, the family of solitary waves is asymptotically linearly stable in $X_{\eta}=L_{\eta}^{2}(\mathbb{R}) \times H_{\eta}^{1}(\mathbb{R})$. More precisely, initial values $\left(n_{0}, v_{0}\right)^{\mathrm{T}} \in(1-P) X_{\eta}$ for $(3.2)$ yield unique solutions $(n(t), v(t))^{\mathrm{T}} \in(1-P) X_{\eta}$ which decay exponentially:

$$
\|(n(t), v(t))\|_{X_{\eta}} \leq C(\eta, \mu) \mathrm{e}^{-\delta(\eta, \mu) t}\left\|\left(n_{0}, v_{0}\right)\right\|
$$

with $C, \delta>0$, independent of $n_{0}, v_{0}$.

Proof. We first have to show that $\mathcal{L}_{*}(\mu)$ generates a strongly continuous semigroup. To see that, it is sufficient to show that $D_{*}=\left(c-v_{*}(x)\right) \partial_{x}$ generates a semigroup on $L_{\eta}^{2}(\mathbb{R})$ and $H_{\eta}^{1}(\mathbb{R})$, since $\mathcal{L}_{*}(\mu)$ can be viewed componentwise as a bounded perturbation of this operator. The operator $D_{*}-\mathrm{id}$ is dissipative, $\operatorname{Re}\left(D_{*} u, u\right)-(u, u) \leq$ 0 , and therefore generates a contraction semigroup. Next, the uniform resolvent estimate from Theorem 2 then ensures that a spectral mapping theorem holds for the linear semigroup (see [2, p. 95]). This proves asymptotic stability as stated.

\section{Essential spectra, point spectra, and the Evans function}

We introduce the basic tools for the spectral analysis in the succeeding sections and prove the statements on the essential spectrum.

Instead of the operator $\mathcal{L}_{*}(\mu)$, we consider the slightly more general operator $L_{*}(\mu)$, obtained from rewriting the eigenvalue problem for the full linearized problem (3.1) as

$$
\begin{aligned}
& \lambda n-c n_{x}+v_{x}+\left(n_{*}(x) v\right)_{x}+\left(v_{*}(x) n\right)_{x}=0, \quad \lambda v-c v_{x}+\psi+\left(v_{*}(x) v\right)_{x}=0, \\
& \varphi_{x}-\psi=0, \quad \psi_{x}+n-\mathrm{e}^{\varphi_{*}(x)} \varphi=0
\end{aligned}
$$

or, in short form, $\left(\lambda B-L_{*}(\mu)\right)(n, v, \varphi, \psi)^{\mathrm{T}}=0$, where $B=\operatorname{diag}(\mathrm{id}, \mathrm{id}, 0,0)$. The operator $L_{*}(\mu)$ is considered as a closed operator on $L^{2} \times H^{1} \times H^{1} \times L^{2}$ with domain $H^{1} \times H^{2} \times H^{2} \times H^{1}$ (from now on we write shortly $L^{2}, H^{1}, \ldots$ instead of $\left.L^{2}(\mathbb{R}), H^{1}(\mathbb{R}), \ldots\right)$.

For the generalized eigenvalue problem (4.1), we define the algebraic multiplicity of the generalized eigenvalue $\lambda$ in case $\lambda B-L_{*}(\mu)$ is Fredholm with index zero as follows. Let $u_{1}^{j} \in \mathbb{R}^{4}, 1 \leq j \leq j_{0}$ be a basis of the kernel and let $u_{\ell}^{j}, 2 \leq \ell \leq \ell_{j}$ be the longest possible generalized Jordan chain $\left(\lambda B-L_{*}(\mu)\right) u_{\ell}^{j}=B u_{\ell-1}^{j}$, for $2 \leq \ell \leq \ell_{j}$, $1 \leq j \leq j_{0}$. The algebraic multiplicity is then defined as the sum of the lengths of the generalized Jordan chains:

$$
\operatorname{alg}(\lambda)=\sum_{j=1}^{j_{0}} \ell_{j} .
$$

The different spectra of the operator $\mathcal{L}_{*}(\mu)$ are found by analyzing the operator $\lambda B-L_{*}(\mu)$. More precisely, the following statements hold. 
Lemma 4.1. Assume $\mu$ is sufficiently small.

(i) The operator $\lambda B-L_{*}(\mu)$ is invertible if and only if $\lambda \notin \operatorname{spec} \mathcal{L}_{*}(\mu)$.

(ii) The operator $\lambda B-L_{*}(\mu)$ is Fredholm with index zero if and only if $\lambda \notin \operatorname{spec}_{\mathrm{ess}} \mathcal{L}_{*}(\mu)$.

(iii) The algebraic multiplicity of $\lambda$ as an eigenvalue of $\mathcal{L}_{*}(\mu)$ and the algebraic multiplicity of $\lambda$ as a generalized eigenvalue to $\lambda B-L_{*}(\mu)$ coincide.

The proofs follow easily from the definitions of these operators, using the invertibility of $\partial_{x x}-\mathrm{e}^{\varphi_{*}}$; we refer to $[1,25]$ for similar statements.

As a first step towards the proofs of Theorems 1 and 2, we characterize essential spectra. Just like in Section 1 (1.3), we compute the dispersion relation to (4.1) from the asymptotic equation at $x= \pm \infty$, setting $n_{*}=v_{*}=\varphi_{*}=0$, with the ansatz $(n, v, \varphi, \psi)=\left(n_{0}, v_{0}, \varphi_{0}, \psi_{0}\right) \mathrm{e}^{v x}$. We find

$$
d(\lambda, v)=\operatorname{det}\left(\begin{array}{cccc}
\lambda-c v & v & 0 & 0 \\
0 & \lambda-c v & 0 & 1 \\
0 & 0 & v & -1 \\
1 & 0 & -1 & v
\end{array}\right)=(\lambda-c v)^{2}\left(v^{2}-1\right)+v^{2}=0
$$

Proposition 4.2. For all $0<\mu<\mu_{0}$ sufficiently small, and any $\eta>0$, the essential spectrum of $\mathcal{L}_{*}(\mu)$ in exponentially weighted spaces $L_{\eta}^{2} \times H_{\eta}^{1}$ is given by the set of $\lambda \in \mathbb{C}$ such that $d(\lambda, v)=0$ for some $v \in \mathbb{C}$ with $\operatorname{Re} v=-\eta$.

Proof. By Lemma 4.1 it is enough to look at the operator $\lambda B-L_{*}(\mu)$. This operator is a differential operator with asymptotically constant coefficients. By setting $n_{*}=v_{*}=\varphi_{*}=0$, from $L_{*}(\mu)$ we find the asymptotic operator $L_{\infty}(\mu)$, at $x= \pm \infty$. Since $\lambda B-L_{*}(\mu)$ is a relatively small perturbation of $\lambda B-L_{\infty}(\mu)$, standard perturbation theory implies that its essential spectrum coincides with the set of $\lambda$ such that $\lambda B-L_{\infty}(\mu)$ is not Fredholm index 0 . The spectrum of $\lambda B-L_{\infty}(\mu)$ is easily analyzed via Fourier transform, which gives the proposition.

Corollary 4.3. For all $0<\mu<\mu_{0}$ sufficiently small, the essential spectrum of $\mathcal{L}_{*}(\mu)$ in $L^{2} \times H^{1}$ is precisely the imaginary axis. In exponentially weighted spaces $L_{\eta}^{2} \times H_{\eta}^{1}$, with $0<\eta<\eta_{0} \mu^{1 / 2}$, the essential spectrum is contained in the set $\{\lambda ; \operatorname{Re} \lambda \leq-\delta(\eta, \mu)<0\}$, for some positive constants $\delta(\eta, \mu)$ and $\eta_{0}$.

Proof. If $d(\lambda, \mathrm{i} k)=0$ for some $k \in \mathbb{R}$, the result in (4.2) gives $\lambda=\mathrm{i} \omega$ with

$$
\omega=c k \pm \frac{k}{\sqrt{k^{2}+1}}
$$

(see Fig. 2). Similarly, in weighted spaces, from $d(\lambda, \mathrm{i} k-\eta)=0$ we find the two branches of the essential spectrum:

$$
\lambda_{ \pm}(k, \eta)=(\mathrm{i} k-\eta)\left(c \pm \frac{1}{\sqrt{1-(\mathrm{i} k-\eta)^{2}}}\right), \quad k \in \mathbb{R} .
$$

These values of $\lambda$ have negative real part and are uniformly bounded away from the imaginary axis, for $c>1$ and $\eta$ sufficiently small. In fact, to first order in $\eta$ we find

$$
\left.\frac{\mathrm{d}(\operatorname{Re} \lambda)}{\mathrm{d} \eta}\right|_{\eta=0}=\left.\frac{\mathrm{d}(\operatorname{Re} \lambda)}{\mathrm{d}(-\operatorname{Re} \nu)}\right|_{\operatorname{Re} \nu=0}=\left.\frac{\mathrm{d}(\operatorname{Im} \lambda)}{\mathrm{d}(-\operatorname{Im} \nu)}\right|_{\operatorname{Re} \nu=0}=-\frac{\mathrm{d} \omega}{\mathrm{d} k}=-c_{\mathrm{g}},
$$



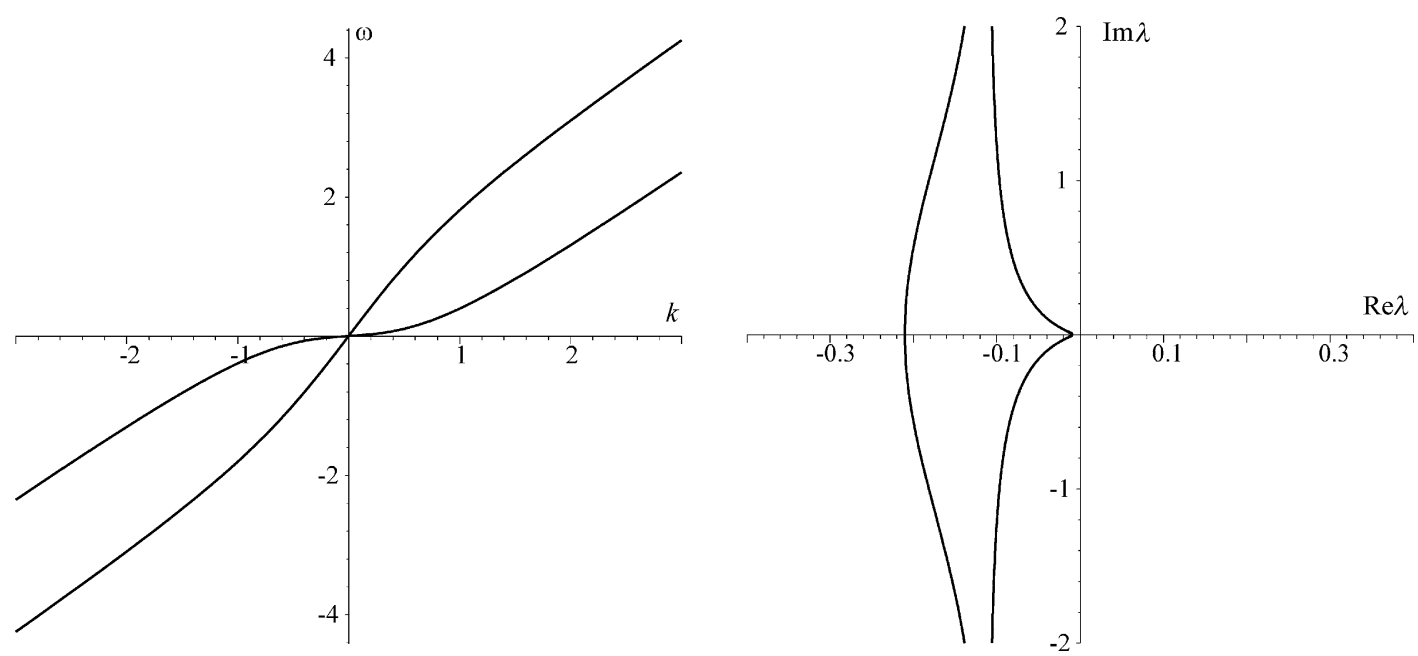

Fig. 2. To the left, the two branches of $\omega(k)$ for $c=1.1$, and to the right, the essential spectrum of $\mathcal{L}_{*}(\mu)$ in exponentially weighted spaces $L_{\eta}^{2} \times H_{\eta}^{1}$, for $c=1.1$ and $\eta=0.1$.

i.e., the essential spectrum moves with the speed of the group velocity in the complex plane when $\eta$ is increased. For $c>1$, supersonic speed, all group velocities are positive, so the essential spectrum moves in the complex left half plane and stabilizes (see Fig. 2). The uniform bound $\operatorname{Re} \lambda \leq-\delta(\eta, \mu)$ is then obtained from the behavior for large $k$ where

$$
\lim _{k \rightarrow \pm \infty} \frac{\mathrm{d}(\operatorname{Re} \lambda)}{\mathrm{d} \eta}(k, \eta)=-c .
$$

Remark 4.4. At sonic speed $c=1$, the essential spectrum of $\mathcal{L}_{*}(\mu)$ coincides with the imaginary axis in $L^{2} \times H^{1}$, and destabilizes in weighted space $L_{\eta}^{2} \times H_{\eta}^{1}$, for any small $\eta>0$, since $\lambda_{-}(0, \eta)=-\eta\left(1-1 / \sqrt{1-\eta^{2}}\right)>0$.

To understand point spectra, it is sufficient to analyze the eigenvalue problem (4.1) and discuss existence of nontrivial, bounded solutions. A very useful tool in this context is the Evans function. Solving the first two equations for $n_{x}$ and $v_{x}$, we find a four-dimensional non-autonomous differential equation:

$$
u_{x}=A(x) u+\lambda M(x) u,
$$

where the matrices $A(x) \rightarrow A_{\infty}, M(x) \rightarrow M_{\infty}$ are asymptotically constant.

The dynamics at $x= \pm \infty$ is described by the eigenvalues $v_{j}, j=1, \ldots, 4$ of $A_{\infty}+M_{\infty}$, which solve the dispersion relation $d\left(\lambda, v_{j}\right)=0$. In particular, for $\operatorname{Re} \lambda>0$, there are precisely three eigenvalues $v_{j}(\lambda), j=1,2,3$ in the complex half plane $\operatorname{Re} v>0$ and one eigenvalue with $\operatorname{Re} v_{4}<0$. For $\operatorname{Re} \lambda=0$, two of these eigenvalues reside on the imaginary axis, $\operatorname{Re} v_{1,2}=0$, and $\operatorname{Re} v_{4}<0<\operatorname{Re} \nu_{3}$. For $|\operatorname{Im} \lambda| \rightarrow+\infty$ and $\mu=0$, we can expand the location of the eigenvalues according to

$$
v_{1,2}(\lambda)=\lambda \pm \mathrm{i}+\mathrm{O}\left(\frac{1}{|\operatorname{Im} \lambda|}\right), \quad v_{3,4}(\lambda)= \pm 1+\mathrm{O}\left(\frac{1}{|\operatorname{Im} \lambda|}\right) .
$$

In the origin, we can expand in $\mu$ and find

$$
v_{1,2}(0)=0, \quad v_{3,4}(0)= \pm \sqrt{\mu}(1+\mathrm{O}(\mu)) .
$$


The 1:3-splitting, $\operatorname{Re} v_{4}(\lambda)<-\eta^{-}(\mu)<-\eta^{+}(\mu)<\operatorname{Re} v_{j}(\lambda), j=1,2,3$, persists for an appropriate choice of $0<\eta^{ \pm}(\mu)=\mathrm{O}\left(\mu^{1 / 2}\right)$ and for all $\operatorname{Re} \lambda \geq-\delta(\mu)$, when $\delta(\mu)>0$ is sufficiently small. Note that this property is strongly related to the fact that for $\mu$ positive the essential spectrum in exponentially weighted spaces moves in the complex left half plane (Corollary 4.3).

For the non-autonomous differential equation, we can then construct a three-dimensional subspace $E_{-}^{\mathrm{u}}(\lambda)$ such that for the solution $u(x ; \lambda)$ to $(4.3)$ with initial value $u(0 ; \lambda)=u_{0}$, we have

$$
u_{0} \in E_{-}^{\mathrm{u}}(\lambda) \Rightarrow|u(x ; \lambda)| \leq C \mathrm{e}^{\eta(\mu)|x|} \quad \text { for all } x<0 .
$$

Similarly, we construct $E_{+}^{\mathrm{s}}(\lambda)$ as the one-dimensional subspace of initial conditions which lead to solutions which decay with exponential rate $\eta(\mu)$, at least, on $x>0$. Clearly, for $\operatorname{Re} \lambda>0$, initial conditions which lead to bounded solutions on $x<0$ lie in $E_{-}^{\mathrm{u}}(\lambda)$ and those which lead to bounded solutions on $x>0$ lie in $E_{+}^{\mathrm{s}}(\lambda)$. In particular, point spectrum in $\operatorname{Re} \lambda>0$ coincides with those values of $\lambda$ such that $E_{-}^{\mathrm{u}}(\lambda)$ and $E_{+}^{\mathrm{s}}(\lambda)$ intersect nontrivially. Choosing analytic bases in the two subspaces $e^{\mathrm{s}}$ and $e_{j}^{\mathrm{u}}, j=1,2,3$, we can detect nontrivial intersections as zeroes of the Evans function:

$$
\mathcal{E}(\lambda ; \mu)=\operatorname{det}\left(e^{\mathrm{s}}, e_{1}^{\mathrm{u}}, e_{2}^{\mathrm{u}}, e_{3}^{\mathrm{u}}\right) .
$$

The function $\mathcal{E}$ is analytic in $\lambda$, for $\operatorname{Re} \lambda>-\delta(\mu)$, and smooth in $\mu$, for sufficiently small $\mu$. The zeroes of this function coincide with the eigenvalues of $\mathcal{L}_{*}(\mu)$ and the order of the zero gives the algebraic multiplicity of the eigenvalues $[1,25]$. The Evans function as constructed above is not unique and merely depends on the choice of bases. However, the zeroes with multiplicity do not depend on that choice.

The above construction is independent of the introduction of small exponential weights, which immediately gives the following lemma.

Lemma 4.5. There exist positive constants $\mu_{0}$ and $\eta_{0}$ such that for all $0<\mu<\mu_{0}$, the point spectra in the unstable complex half plane $\operatorname{Re} \lambda>0$ of $\mathcal{L}_{*}(\mu)$, considered in $L^{2} \times H^{1}$ or in the weighted space $L_{\eta}^{2} \times H_{\eta}^{1}$, coincide with multiplicity for $0<\eta<\eta_{0} \mu^{1 / 2}$. Moreover, for $0<\eta<\eta^{\prime}<\eta_{0} \mu^{1 / 2}$, the point spectra in $L_{\eta}^{2} \times H_{\eta}^{1}$ and $L_{\eta^{\prime}}^{2} \times H_{\eta^{\prime}}^{1}$ coincide with multiplicity in a region $\operatorname{Re} \lambda>-\delta(\eta, \mu)$, for $\delta(\eta, \mu)>0$ sufficiently small.

In particular, this lemma shows that, given the results on essential spectra in Corollary 4.3, Theorem 1 is a consequence of Theorem 2, since the point spectrum of $\mathcal{L}_{*}(\mu)$ is symmetric with respect to the imaginary axis due to reversibility (reflect $t \rightarrow-t, x \rightarrow-x$, and use the fact that the solitary wave is an even function).

In the succeeding sections, we prove the remaining claims of Theorem 2 on the point spectrum in exponentially weighted spaces and the resolvent estimates.

\section{Non-zero and high frequencies}

We show that there are no eigenvalues in a region $|\lambda| \geq \varepsilon_{0}, \operatorname{Re} \lambda \geq-\delta(\mu, \eta)$, for sufficiently small $\mu$, sufficiently small weight $\eta$, and any $\varepsilon_{0}>0$. Moreover we prove the resolvent estimates from Theorem 2 .

We exclude eigenvalues in a region $|\lambda| \geq \varepsilon_{0}, \operatorname{Re} \lambda>-\delta(\eta, \mu)$ by inverting the operator $\lambda$ id $-\mathcal{L}_{*}(\mu)$ in the weighted space $X_{\eta}=L_{\eta}^{2} \times H_{\eta}^{1}$. We do this by solving the system

$$
\begin{aligned}
& \lambda n+\left(-c+v_{*}(x)\right)\left(n_{x}-\eta n\right)+\left(1+n_{*}(x)\right)\left(v_{x}-\eta v\right)+v_{*}^{\prime}(x) n+n_{*}^{\prime}(x) v=f_{n}, \\
& \lambda v+\left(-c+v_{*}(x)\right)\left(v_{x}-\eta v\right)+\psi+v_{*}^{\prime}(x) v=f_{v}, \\
& \varphi_{x}-\eta \varphi-\psi=0, \quad \psi_{x}-\eta \psi+n-\mathrm{e}^{\varphi_{*}(x)} \varphi=0
\end{aligned}
$$


in $L^{2} \times H^{1} \times H^{1} \times L^{2}$, for $\left(f_{n}, f_{v}\right) \in L^{2} \times H^{1}$. Estimates on the norms of $n$ and $v$ will give the desired resolvent estimates.

For small $\mu$, the left hand side of (5.1) defines a linear operator which is a relatively bounded perturbation of the operator with constant coefficients, at $\mu=0$. After a change of variables that we introduce now, the perturbation becomes bounded in the operator norm.

We define $\xi(x)$ through

$$
\xi^{\prime}(x)=\left(c-v_{*}(x)\right)^{-1}, \quad \xi(0)=0 .
$$

For $\mu$ sufficiently small, $\xi(x)$ gives a valid change of variable, inducing equivalent $L^{2}$-norms. The system (5.1) becomes (we write again $x$ instead of $\xi$ )

$$
\begin{aligned}
& \lambda n-\left(n_{x}-\eta n\right)+\left(v_{x}-\eta v\right)+\mathrm{O}(\mu)=f_{n}, \quad \lambda v-\left(v_{x}-\eta v\right)+\psi+\mathrm{O}(\mu)=f_{v}, \\
& \varphi_{x}-\eta \varphi-\psi+\mathrm{O}(\mu)=0, \quad \psi_{x}-\eta \psi+n-\varphi+\mathrm{O}(\mu)=0,
\end{aligned}
$$

where $\mathrm{O}(\mu)$ denotes a small, bounded perturbation:

$$
\tilde{L}_{1}(\mu, \eta): L^{2} \times H^{1} \times H^{1} \times L^{2} \rightarrow L^{2} \times H^{1} \times H^{1} \times L^{2}, \quad\left\|\tilde{L}_{1}(\mu, \eta)\right\| \leq C \mu .
$$

We first analyze the autonomous system at $\mu=0$, and then, by a perturbation argument, the full system, for sufficiently small $\mu>0$. At $\mu=0$ the left hand side in(5.2) defines a differential operator $\tilde{L}_{0}(\lambda, \eta)$, with constant coefficients, on $L^{2} \times H^{1} \times H^{1} \times L^{2}$ with domain $H^{1} \times H^{2} \times H^{2} \times H^{1}$.

Lemma 5.1. For any $\varepsilon_{0}>0$ there are positive constants $\eta_{0}$ and $C_{0}$ such that for all $\eta \in\left(0, \eta_{0}\right)$ and all $\lambda$ with $\operatorname{Re} \lambda+\eta / 2>0,|\lambda| \geq \varepsilon_{0}$, the operator $\tilde{L}_{0}(\lambda, \eta)$ has a bounded inverse in $L^{2} \times H^{1} \times H^{1} \times L^{2}$ with norm

$$
\left\|\tilde{L}_{0}(\lambda, \eta)\right\| \leq \frac{C_{0}}{\operatorname{Re} \lambda+\eta} .
$$

Proof. We invert $\tilde{L}_{0}(\lambda, \eta)$ by solving the system

$$
\begin{aligned}
& \lambda n-\left(n_{x}-\eta n\right)+\left(v_{x}-\eta v\right)=f_{n}, \quad \lambda v-\left(v_{x}-\eta v\right)+\psi=f_{v}, \\
& \varphi_{x}-\eta \varphi-\psi=f_{\varphi}, \quad \psi_{x}-\eta \psi+n-\varphi=f_{\psi}
\end{aligned}
$$

in $L^{2} \times H^{1} \times H^{1} \times L^{2}$. By taking the Fourier transform in $x$ we find a linear system that we can solve explicitly and obtain

$$
\begin{aligned}
& {\left[(\lambda+\eta-\mathrm{i} k)^{2}-\frac{(\mathrm{i} k-\eta)^{2}}{1-(\mathrm{i} k-\eta)^{2}}\right] \hat{n}=(\lambda+\eta-\mathrm{i} k) \hat{f}_{n}-(\mathrm{i} k-\eta) g_{v},} \\
& {\left[(\lambda+\eta-\mathrm{i} k)^{2}-\frac{(\mathrm{i} k-\eta)^{2}}{1-(\mathrm{i} k-\eta)^{2}}\right] \hat{v}=(\lambda+\eta-\mathrm{i} k) g_{v}-\frac{\mathrm{i} k-\eta}{1-(\mathrm{i} k-\eta)^{2}} \hat{f}_{n},} \\
& \hat{\varphi}=\frac{\hat{n}-\hat{f}_{\psi}-(\mathrm{i} k-\eta) \hat{f}_{\varphi}}{1-(\mathrm{i} k-\eta)^{2}}, \quad \hat{\psi}=(\mathrm{i} k-\eta) \hat{\varphi}-\hat{f}_{\varphi},
\end{aligned}
$$

where

$$
g_{v}=\hat{f}_{v}+\frac{\hat{f}_{\varphi}-(\mathrm{i} k-\eta) \hat{f}_{\psi}}{1-(\mathrm{i} k-\eta)^{2}} \in H^{1} .
$$


For any $|\lambda|>\varepsilon_{0}, \operatorname{Re} \lambda+\eta / 2>0$, and sufficiently small $\eta \in\left(0, \eta_{0}\right)$, we show that

$$
\left|(\lambda+\eta-\mathrm{i} k)^{2}-\frac{(\mathrm{i} k-\eta)^{2}}{1-(\mathrm{i} k-\eta)^{2}}\right|^{2} \geq C\left(\varepsilon_{0}\right)(\operatorname{Re} \lambda+\eta)^{2}\left(1+(\operatorname{Re} \lambda+\eta)^{2}+(\operatorname{Im} \lambda-k)^{2}\right) .
$$

This lower bound together with the explicit formulas above proves the lemma.

We obtain (5.4) first in a region $\operatorname{Re} \lambda>\varepsilon_{1}$, and then for $\operatorname{Im} \lambda>\varepsilon_{1}$, for any $\varepsilon_{1}>0$. A direct calculation gives

$$
\begin{aligned}
\left|(\lambda+\eta-\mathrm{i} k)^{2}-\frac{(\mathrm{i} k-\eta)^{2}}{1-(\mathrm{i} k-\eta)^{2}}\right|^{2}= & (\operatorname{Re} \lambda+\eta)^{4}+(\operatorname{Im} \lambda-k)^{4}+2(\operatorname{Re} \lambda+\eta)^{2}(\operatorname{Im} \lambda-k)^{2}+b_{1}^{2} \\
& +2(\operatorname{Re} \lambda+\eta)^{2} b_{1}-2(\operatorname{Im} \lambda-k)^{2} b_{1}+4 b_{2}^{2}+8(\operatorname{Re} \lambda+\eta)(\operatorname{Im} \lambda-k) b_{2},
\end{aligned}
$$

where

$$
b_{1}=\frac{\left(k^{2}+\eta^{2}\right)^{2}+k^{2}-\eta^{2}}{\left(1+k^{2}-\eta^{2}\right)^{2}+4 k^{2} \eta^{2}} \in\left[-\frac{\eta^{2}}{1-\eta^{2}}, 1\right], \quad b_{2}=\frac{k \eta}{\left(1+k^{2}-\eta^{2}\right)^{2}+4 k^{2} \eta^{2}} \in\left[-c_{1} \eta, c_{1} \eta\right]
$$

for some positive constant $c_{1}$ which does not depend upon $\eta$.

For $\operatorname{Re} \lambda>\varepsilon_{1}$, the right hand side in (5.5) is bounded below by

$$
\begin{aligned}
& (\operatorname{Re} \lambda+\eta)^{4}+(\operatorname{Re} \lambda+\eta)^{2}(\operatorname{Im} \lambda-k)^{2}-2(\operatorname{Re} \lambda+\eta)^{2} \frac{\eta^{2}}{1-\eta^{2}}-12 b_{2}^{2} \\
& \geq \frac{1}{2}(\operatorname{Re} \lambda+\eta)^{4}+(\operatorname{Re} \lambda+\eta)^{2}(\operatorname{Im} \lambda-k)^{2}+\frac{\varepsilon_{1}^{4}}{4}-\frac{4 \eta^{4}}{\left(1-\eta^{2}\right)^{2}}-12 c_{1}^{2} \eta^{2},
\end{aligned}
$$

so (5.4) holds for sufficiently small $\eta$.

Assume now $|\operatorname{Im} \lambda|>\varepsilon_{1}$. For $|\operatorname{Im} \lambda-k| \leq \delta$, with $\delta$ sufficiently small, we have $|k| \geq \varepsilon_{1} / 2$ and $b_{1} \geq b_{1}\left(\varepsilon_{1}\right)>0$. Then the right hand side in (5.5) is bounded below by

$$
\begin{aligned}
&(\operatorname{Re} \lambda+\eta)^{4}+(\operatorname{Re} \lambda+\eta)^{2}(\operatorname{Im} \lambda-k)^{2}+b_{1}^{2}+2(\operatorname{Re} \lambda+\eta)^{2} b_{1}-2 \delta^{2} b_{1}-12 b_{2}^{2} \\
& \geq(\operatorname{Re} \lambda+\eta)^{4}+(\operatorname{Re} \lambda+\eta)^{2}(\operatorname{Im} \lambda-k)^{2}+2 b_{1}\left(\varepsilon_{1}\right)(\operatorname{Re} \lambda+\eta)^{2} \\
& \quad+\frac{1}{2} b_{1}\left(b_{1}\left(\varepsilon_{1}\right)-4 \delta^{2}\right)+\frac{1}{2}\left(b_{1}\left(\varepsilon_{1}\right)^{2}-24 c_{1}^{2} \eta^{2}\right)
\end{aligned}
$$

and (5.4) holds for sufficiently small $\eta$ and $\delta\left(\varepsilon_{1}\right)$.

If $|\operatorname{Im} \lambda-k| \geq \delta\left(\varepsilon_{1}\right)$, we consider first $|k| \geq k_{*} \eta$. Then the right hand side in (5.5) is bounded below by

$$
\begin{gathered}
(\operatorname{Re} \lambda+\eta)^{4}+(\operatorname{Re} \lambda+\eta)^{2}(\operatorname{Im} \lambda-k)^{2}+2(\operatorname{Re} \lambda+\eta)^{2} b_{1}-12 b_{2}^{2} \\
\geq(\operatorname{Re} \lambda+\eta)^{4}+(\operatorname{Re} \lambda+\eta)^{2}(\operatorname{Im} \lambda-k)^{2}+\frac{1}{2}\left(\eta^{2}\right) b_{1}-12 b_{2}^{2} .
\end{gathered}
$$

For $k_{*}$ large enough we have $24 b_{2}^{2}<b_{1} \eta^{2}$, and (5.4) follows since $|\operatorname{Im} \lambda-k| \geq \delta\left(\varepsilon_{1}\right)$. Next, if $|k| \leq k_{*} \eta$, we find the lower bound

$$
\begin{aligned}
& (\operatorname{Re} \lambda+\eta)^{4}+(\operatorname{Im} \lambda-k)^{4}+(\operatorname{Re} \lambda+\eta)^{2}(\operatorname{Im} \lambda-k)^{2}+2(\operatorname{Re} \lambda+\eta)^{2} b_{1}-2(\operatorname{Im} \lambda-k)^{2} b_{1}-12 b_{2}^{2} \\
& \geq(\operatorname{Re} \lambda+\eta)^{4}+\frac{1}{2}(\operatorname{Re} \lambda+\eta)^{2}(\operatorname{Im} \lambda-k)^{2}+\frac{1}{2}(\operatorname{Re} \lambda+\eta)^{2}\left(\delta\left(\varepsilon_{1}\right)^{2}+4 b_{1}\right) \\
& \quad+\frac{1}{2}(\operatorname{Im} \lambda-k)^{2}\left(\delta\left(\varepsilon_{1}\right)^{2}-4 b_{1}\right)+\frac{1}{2} \delta\left(\varepsilon_{1}\right)^{4}-12 c_{1}^{2} \eta^{2}
\end{aligned}
$$

from which we obtain (5.4), for sufficiently small $\eta$, since $\left|b_{1}\right| \leq c_{2} \eta^{2}$, for $|k| \leq k_{*} \eta$. This completes the proof of the inequality (5.4). 
Corollary 5.2. For all $\varepsilon_{0}>0$ there exists $\eta_{0}>0$ and $\mu_{0}>0$ such that, for all $\eta \in\left(0, \eta_{0}\right), \mu \in\left(0, \mu_{0} \eta\right)$ and $|\lambda| \geq \varepsilon_{0}, \operatorname{Re} \lambda>-\eta / 2$ the operator $\lambda \mathrm{id}-\mathcal{L}_{*}(\mu)$ has a bounded inverse in the weighted space $X_{\eta}=L_{\eta}^{2} \times H_{\eta}^{1}$. Moreover, we have the estimate on the resolvent

$$
\left\|\lambda \operatorname{id}-\mathcal{L}_{*}(\mu)\right\|_{X_{\eta} \rightarrow X_{\eta}} \leq \frac{C}{\operatorname{Re} \lambda+\eta}
$$

for some positive constant $C$ depending upon $\varepsilon_{0}$.

Proof. By Lemma 5.1 we can choose $\mu_{0}$ sufficiently small such that

$$
\left\|\tilde{L}_{0}(\lambda, \eta)^{-1} \tilde{L}_{1}(\mu, \eta)\right\| \leq 2 C_{0} C \mu_{0} \leq \frac{1}{2}
$$

and we conclude that the operator $\tilde{L}_{0}(\lambda, \eta)+\tilde{L}_{1}(\mu, \eta)$ is invertible, so $\lambda$ id $-\mathcal{L}_{*}(\mu)$ is invertible as well.

This corollary, together with Lemma 4.5, proves the statements in Theorem 2, outside any neighborhood of the origin $|\lambda|<\varepsilon_{0}$.

\section{The zero-frequency limit and the Korteweg-de Vries scaling}

The goal of this section is to analyze the linearization in a neighborhood of $\lambda=0$. In a suitable scaling, we find, at the lowest order in $\mu$, the Korteweg-de Vries equation, for which the spectra in an exponentially weighted space is exactly known. Critical and unstable spectrum consists precisely of one double eigenvalue in the origin. Since this double eigenvalue is induced by spatial translation and the Galilean invariance, it is robust and cannot create instabilities after perturbation. The analysis in this section is very much reminiscent of [14,27].

The outline of this section is as follows. In Section 6.1, we review the dispersion relation in a small neighborhood of $\lambda=v=0$. We then expand the linearized system in the perturbation parameter $\mu$ and transform variables to find the Jordan normal form at $\mu=0$. In Section 6.2, we scale variables to find the linearization about the Korteweg-de Vries soliton to leading order as a subsystem of our four-dimensional linear equation. We compute the Evans function of the full system and show that it is robust with respect to higher order perturbations. In the last paragraph, Section 6.3, we finally justify the Korteweg-de Vries scaling proving absence of eigenvalues in a small neighborhood of the origin, outside this scaling.

\subsection{Expanding the linearization near $\lambda=0$}

The dispersion relation at the asymptotic state of the solitary wave was computed as $d(\lambda, v)=(\lambda-c \nu)^{2}\left(v^{2}-1\right)$ $+v^{2}=0$ with $c^{2}=1+\mu$. For $\mu=0$, we find a quadruple root $v=0$ at $\lambda=0$, which unfolds like

$$
v_{0}=\frac{1}{2} \lambda+\mathrm{O}\left(|\lambda|^{3}\right), \quad v_{1,2,3}=-(2 \lambda)^{1 / 3}+\mathrm{O}(|\lambda|)
$$

The three eigenvalues $v_{1,2,3}$ stem from the Korteweg-de Vries limit, whereas $v_{0}$ introduces an additional root of the dispersion relation, with a non-zero group velocity.

For $\mu>0$ and $\operatorname{Re} \lambda \geq 0$, we find three roots in the right half plane $\operatorname{Re} v \geq 0$ and one root in the left half plane $\operatorname{Re} v<0$. This fact will allow us to construct an Evans function in a robust way.

Remark 6.1. Surprisingly enough, the fourth eigenvalue $v_{0}$ seems to appear in all known examples, where solitary waves have been found in a Korteweg-de Vries limit of a physically realistic system. The group velocity associated 
with this root can however be positive as well as negative. Within the Korteweg-de Vries scaling, transport is always unidirectional - the central observation in [25,26] which allowed for a proof of asymptotic stability using exponentially weighted spaces. The transport induced by the fourth eigenvalue happens to be in the same direction in the plasma model, as well as in model equations for water-waves [27] or the full water-wave problem without surface tension [14]. However, for gravity surface waves in the presence of large surface tension, the group velocities from the additional branch of the dispersion relation have the opposite sign! As a consequence, the proof of stability in [14] is, in consequence, much more subtle.

We expand the linearized system (4.1) in a neighborhood of the origin, solving for $n_{x}$ and $v_{x}$ :

$$
\begin{aligned}
& n_{x}=\lambda n+v_{*}^{\prime} n+\lambda v+\left(v_{*}^{\prime}+n_{*}^{\prime}\right) v+\psi+\left(-\mu+n_{*}+2 v_{*}\right) \psi+\mathrm{O}\left(\left(|\lambda \mu|+\mu^{5 / 2}\right)(|n|+|v|)+\mu^{2}|\psi|\right) \\
& v_{x}=\lambda v+v_{*}^{\prime} v+\psi+\left(-\frac{1}{2} \mu+v_{*}\right) \psi+\mathrm{O}\left(\left(|\lambda \mu|+\mu^{5 / 2}\right)|v|+\mu^{2}|\psi|\right) \\
& \varphi_{x}=\psi, \quad \psi_{x}=\varphi-n+\left(\mathrm{e}^{\varphi_{*}}-1\right) \varphi
\end{aligned}
$$

and transform variables to put the linear part at $\mu=\lambda=0$ into Jordan normal form. We set

$$
n=a_{1}, \quad v=a_{0}+a_{1}, \quad \varphi=a_{1}+a_{3}, \quad \psi=a_{2} .
$$

The system for $\left(a_{0}, a_{1}, a_{2}, a_{3}\right) \in \mathbb{C}^{4}$ becomes

$$
\begin{aligned}
a_{0, x}= & -\lambda a_{1}-\mu^{3 / 2} \Phi_{*}^{\prime} a_{0}-2 \mu^{3 / 2} \Phi_{*}^{\prime} a_{1}+\mu\left(\frac{1}{2}-2 \Phi_{*}\right) a_{2}+\mathrm{O}\left(\left(|\lambda \mu|+\mu^{5 / 2}\right)\left(\left|a_{0}\right|+\left|a_{1}\right|\right)+\mu^{2}\left|a_{2}\right|\right), \\
a_{1, x}= & a_{2}+\lambda\left(a_{0}+2 a_{1}\right)+2 \mu^{3 / 2} \Phi_{*}^{\prime} a_{0}+3 \mu^{3 / 2} \Phi_{*}^{\prime} a_{1}-\mu\left(1-3 \Phi_{*}\right) a_{2} \\
& +\mathrm{O}\left(\left(|\lambda \mu|+\mu^{5 / 2}\right)\left(\left|a_{0}\right|+\left|a_{1}\right|\right)+\mu^{2}\left|a_{2}\right|\right) \\
a_{2, x}= & a_{3}+\mu \Phi_{*}\left(a_{1}+a_{3}\right)+\mathrm{O}\left(\mu^{2}\left(\left|a_{1}\right|+\left|a_{3}\right|\right)\right) \\
a_{3, x}= & -\lambda\left(a_{0}+2 a_{1}\right)-2 \mu^{3 / 2} \Phi_{*}^{\prime} a_{0}-3 \mu^{3 / 2} \Phi_{*}^{\prime} a_{1}+\mu\left(1-3 \Phi_{*}\right) a_{2} \\
& +\mathrm{O}\left(\left(|\lambda \mu|+\mu^{5 / 2}\right)\left(\left|a_{0}\right|+\left|a_{1}\right|\right)+\mu^{2}\left|a_{2}\right|\right)
\end{aligned}
$$

where we used that, by (2.3),

$$
\left(n_{*}, v_{*}, \mathrm{e}^{\varphi_{*}}-1\right)(x)=\mu \Phi_{*}(\sqrt{\mu} x)(1,1,1)+\mathrm{O}\left(\mu^{2}\right) .
$$

\subsection{The Korteweg-de Vries scaling}

The natural scaling, suggested from the dispersion relation, is

$$
x=\mu^{1 / 2} \xi, \quad \lambda=\mu^{3 / 2} \Lambda, \quad a_{0}=\mu b_{0}, \quad a_{1}=b_{1}, \quad a_{2}=\mu^{1 / 2} b_{2}, \quad a_{3}=\mu b_{3} .
$$

The system (6.2) reads

$$
\begin{aligned}
& b_{0, \xi}=-\Lambda b_{1}-2 \Phi_{*}^{\prime} b_{1}+\left(\frac{1}{2}-2 \Phi_{*}\right) b_{2}+\mathrm{O}(\mu), \quad b_{1, \xi}=b_{2}+\mathrm{O}(\mu), \quad b_{2, \xi}=b_{3}+\Phi_{*} b_{1}+\mathrm{O}(\mu) \\
& b_{3, \xi}=-2 \Lambda b_{1}-3 \Phi_{*}^{\prime} b_{1}+\left(1-3 \Phi_{*}\right) b_{2}+\mathrm{O}(\mu)
\end{aligned}
$$

At $\mu=0$, we find the linearization about the Korteweg-de Vries soliton

$$
b_{1, \xi \xi \xi}=-2 \Lambda b_{1}+b_{1, \xi}-2\left(\Phi_{*} b_{1}\right) \xi
$$

decoupled from the equation for $b_{0}$. 
As a next step, we construct an Evans function, analytic in $\Lambda$ and smooth in $\mu$. First, we analyze the dispersion relation to (6.3) at the asymptotic state, $\xi= \pm \infty$, and $\mu=0$. Setting $b_{j}(\xi)=b_{j 0} \mathrm{e}^{\vartheta \xi}$, we find

$$
d_{\text {sca }}(\Lambda, \vartheta)=\operatorname{det}\left(\begin{array}{cccc}
\vartheta & \Lambda & -\frac{1}{2} & 0 \\
0 & \vartheta & -1 & 0 \\
0 & 0 & \vartheta & -1 \\
0 & 2 \Lambda & -1 & \vartheta
\end{array}\right)=\vartheta\left(\vartheta^{3}-\vartheta+2 \Lambda\right)=\vartheta d_{\mathrm{KdV}}(\Lambda, \vartheta)
$$

The four eigenvalues $\vartheta_{j}$, satisfying $d_{\text {sca }}\left(\Lambda, \vartheta_{j}\right)=0$, are

$$
\vartheta_{1}=0, \quad \vartheta_{2}=2 \Lambda+\mathrm{O}(\Lambda), \quad \vartheta_{3}=1+\mathrm{O}(\Lambda), \quad \vartheta_{4}=-1+\mathrm{O}(\Lambda) .
$$

For sufficiently small $\operatorname{Re} \Lambda \geq-\delta_{0}$, they are separated by $\operatorname{Re} \vartheta_{4}<-\tilde{\eta}<\operatorname{Re} \vartheta_{1,2,3}$, for some $\tilde{\eta} \in(0,1)$. This splitting persists for any $\mu$ sufficiently small, so we can construct an Evans function $\mathcal{E}_{\text {sca }}(\Lambda ; \mu)$, analytic in $\Lambda$ and smooth in $\mu$ (see Section 4).

Lemma 6.2. For any constant $M>0$ there is $\delta>0$ such that for $\operatorname{Re} \Lambda \geq-\delta$ and $|\Lambda|<M$

$$
\mathcal{E}_{\mathrm{sca}}(\Lambda ; 0)=\Lambda^{2}+\mathrm{O}\left(\Lambda^{3}\right) \text { as } \Lambda \rightarrow 0
$$

and

$$
\mathcal{E}_{\text {sca }}(\Lambda ; 0) \neq 0, \quad \text { if } \Lambda \neq 0
$$

Proof. We compute an Evans function for the scaled system (6.3) at $\mu=0$. A trivial solution of the equation for $\mu=$ 0 is given by $b^{0}(\xi) \equiv(1,0,0,0)^{\mathrm{T}}$. The equations for $b_{1}, b_{2}, b_{3}$ are independent of $b_{0}$ and consist of the linearization about the Korteweg-de Vries soliton. They possess solutions $b_{\mathrm{KdV}}^{\mathrm{s}}(\xi ; \Lambda), b_{\mathrm{KdV}}^{\mathrm{u}, 1}(\xi ; \Lambda)$, and $b_{\mathrm{KdV}}^{\mathrm{u}, 2}(\xi ; \Lambda)$ such that

$$
\begin{aligned}
& \left|b_{\mathrm{KdV}}^{\mathrm{s}}(\xi ; \Lambda)\right| \leq C \mathrm{e}^{\operatorname{Re} \vartheta_{4} \xi} \leq C \mathrm{e}^{-\tilde{\eta}|\xi|} \quad \text { for } \xi>0, \quad\left|b_{\mathrm{KdV}}^{\mathrm{u}, 1}(\xi ; \Lambda)\right| \leq C \mathrm{e}^{\operatorname{Re} \vartheta_{3} \xi} \leq C \mathrm{e}^{\tilde{\eta}|\xi|} \quad \text { for } \xi<0, \\
& \left|b_{\mathrm{KdV}}^{\mathrm{u}, 2}(\xi ; \Lambda)\right| \leq C \mathrm{e}^{\operatorname{Re} \vartheta_{2} \xi} \leq C \mathrm{e}^{\tilde{\eta}|\xi|} \text { for } \xi<0 .
\end{aligned}
$$

We define $\mathcal{E}_{\mathrm{KdV}}(\Lambda):=\operatorname{det}\left(b_{\mathrm{KdV}}^{\mathrm{s}}(0 ; \Lambda), b_{\mathrm{KdV}}^{\mathrm{u}, 1}(0 ; \Lambda), b_{\mathrm{KdV}}^{\mathrm{u}, 2}(0 ; \Lambda)\right)$. From [25], we conclude that $\mathcal{E}_{\mathrm{KdV}}(\Lambda) \neq 0$ for $\Lambda \neq 0$ and $\mathcal{E}_{\mathrm{KdV}}(\Lambda)=\Lambda^{2}+\mathrm{O}\left(\Lambda^{3}\right)$, as $\Lambda \rightarrow 0$. From the three solutions $b_{\mathrm{KdV}}^{j}$, we find solutions of the full scaled system (6.3), at $\mu=0$, from simple integration of the equation for $b_{0, \xi}$. For $\operatorname{Re} \Lambda>0$, we set

$$
\begin{aligned}
& b_{0}^{\mathrm{s}}(\xi ; \Lambda)=\int_{\infty}^{\xi}\left(-\Lambda b_{1, \mathrm{KdV}}^{\mathrm{s}}(\zeta)-2 \Phi_{*}^{\prime}(\zeta) b_{1, \mathrm{KdV}}^{\mathrm{s}}(\zeta)-2 \Phi_{*}(\zeta) b_{2, \mathrm{KdV}}^{\mathrm{s}}(\zeta)\right) \mathrm{d} \zeta+\frac{1}{2} b_{1, \mathrm{KdV}}^{\mathrm{s}}(\xi), \\
& b_{0}^{\mathrm{u}, 1}(\xi ; \Lambda)=\int_{-\infty}^{\xi}\left(-\Lambda b_{1, \mathrm{KdV}}^{\mathrm{u}, 1}(\zeta)-2 \Phi_{*}^{\prime}(\zeta) b_{1, \mathrm{KdV}}^{\mathrm{u}, 1}(\zeta)-2 \Phi_{*}(\zeta) b_{2, \mathrm{KdV}}^{\mathrm{u}, 1}(\zeta)\right) \mathrm{d} \zeta+\frac{1}{2} b_{1, \mathrm{KdV}}^{\mathrm{u}, 1}(\xi), \\
& b_{0}^{\mathrm{u}, 2}(\xi ; \Lambda)=\int_{-\infty}^{\xi}\left(-\Lambda b_{1, \mathrm{KdV}}^{\mathrm{u}, 2}(\zeta)-2 \Phi_{*}^{\prime}(\zeta) b_{1, \mathrm{KdV}}^{\mathrm{u}, 2}(\zeta)-2 \Phi_{*}(\zeta) b_{2, \mathrm{KdV}}^{\mathrm{u}, 2}(\zeta)\right) \mathrm{d} \zeta+\frac{1}{2} b_{1, \mathrm{KdV}}^{\mathrm{u}, 2}(\xi)
\end{aligned}
$$

Note that the integrals share the exponential decay estimates for the vectors $b_{\mathrm{KdV}}^{j}$ (6.4). The vectors $b_{\mathrm{sca}}^{j}=$ $\left(b_{0}^{j}(0), b_{1, \mathrm{KdV}}^{j}(0), b_{2, \mathrm{KdV}}^{j}(0), b_{3, \mathrm{KdV}}^{j}(0)\right)^{\mathrm{T}}$, with $j=\mathrm{u}, 1$ or $j=\mathrm{u}, 2$, together with $b^{0}=(1,0,0,0)$ form a basis of the unstable subspace at $\xi=0$ of the scaled system, and $b_{\text {sca }}^{\mathrm{s}}=\left(b_{0}^{\mathrm{s}}(0), b_{1, \mathrm{KdV}}^{\mathrm{s}}(0), b_{2, \mathrm{KdV}}^{\mathrm{s}}(0), b_{3, \mathrm{KdV}}^{\mathrm{s}}(0)\right)^{\mathrm{T}}$ 
spans the stable subspace. An Evans function for the scaled system at $\mu=0$, for $\operatorname{Re} \Lambda>0$, is therefore found from

$$
\mathcal{E}_{\text {sca }}(\Lambda ; 0):=\operatorname{det}\left(b^{0}, \quad b_{\text {sca }}^{\mathrm{s}}, \quad b_{\text {sca }}^{\mathrm{u}, 1}, \quad b_{\text {sca }}^{\mathrm{u}, 2}\right)=\operatorname{det}\left(\begin{array}{cccc}
1 & b_{0}^{\mathrm{u}, 1}(0) & b_{0}^{\mathrm{u}, 2}(0) & b_{0}^{\mathrm{s}}(0) \\
0 & b_{1, \mathrm{KdV}}^{\mathrm{u}, 1}(0) & b_{1, \mathrm{KdV}}^{\mathrm{u}, 2}(0) & b_{1, \mathrm{KdV}}^{\mathrm{s}}(0) \\
0 & b_{2, \mathrm{KdV}}^{\mathrm{u}, 1}(0) & b_{2, \mathrm{KdV}}^{\mathrm{u}, 2}(0) & b_{2, \mathrm{KdV}}^{\mathrm{s}}(0) \\
0 & b_{3, \mathrm{KdV}}^{\mathrm{u}, 1}(0) & b_{3, \mathrm{KdV}}^{\mathrm{u}, 2}(0) & b_{3, \mathrm{KdV}}^{\mathrm{s}}(0)
\end{array}\right)=\mathcal{E}_{\mathrm{KdV}}(\Lambda) .
$$

The analyticity in $\Lambda$ shows that $\mathcal{E}_{\text {sca }}(\Lambda ; 0)=\mathcal{E}_{\mathrm{KdV}}(\Lambda)$, for $\operatorname{Re} \Lambda>-\delta$, which proves the lemma.

Next, note that $\mathcal{E}_{\text {sca }}(0 ; \mu)=\mathcal{E}_{\text {sca }}^{\prime}(0 ; \mu)=0$ even for $\mu \geq 0$, since an eigenvector and a generalized eigenfunction are provided by the derivative of the solitary wave with respect to space $x$ and speed $c$, respectively. Continuity in $\mu$ and analyticity in $\Lambda$ guarantee that no additional zeroes besides the double zero at the origin may emerge for small $\mu$. This proves the absence of point spectrum in regions $|\lambda| \leq M \mu^{3 / 2}, \operatorname{Re} \lambda \geq-\delta \mu^{3 / 2}$, besides the double eigenvalue at the origin.

\subsection{Justifying the Korteweg-de Vries scaling}

We conclude the proof of Theorem 2 showing absence of eigenvalues in a small neighborhood of the origin. We may therefore assume that $\mu=\delta|\lambda|^{2 / 3}$, with $\delta$ small. We may scale

$$
\xi=|\lambda|^{1 / 3} x, \quad a_{0}=|\lambda|^{2 / 3} b_{0}, \quad a_{1}=b_{1}, \quad a_{2}=|\lambda|^{1 / 3} b_{2}, \quad a_{3}=|\lambda|^{2 / 3} b_{3} .
$$

In scaled coordinates we find

$$
\begin{aligned}
& b_{0, \xi}=-\mathrm{e}^{\mathrm{i} \arg \lambda} b_{1}+\mathrm{O}(|\delta|), \quad b_{1, \xi}=b_{2}+\mathrm{O}\left(|\lambda|^{2 / 3}\right), \quad b_{2, \xi}=b_{3}+\mathrm{O}(|\delta|), \\
& b_{3, \xi}=-2 \mathrm{e}^{\mathrm{i} \arg \lambda} b_{1}+\mathrm{O}\left(|\delta|+|\lambda|^{2 / 3}\right) .
\end{aligned}
$$

Setting $\delta=\lambda=0$, we find an autonomous linear equation with eigenvalues $\tilde{v}$ satisfying

$$
\tilde{v}^{4}+2 \mathrm{e}^{\mathrm{i} \arg \lambda} \tilde{v}=0,
$$

i.e.:

$$
\tilde{v}_{0}=0, \quad \tilde{v}_{2,3,4}^{3}=-2 \mathrm{e}^{\mathrm{i} \arg \lambda} .
$$

Again, for $\arg \lambda \neq \pi$, the eigenvalues are well separated by a spectral gap $\tilde{v}_{4}<\tilde{\eta}<\tilde{v}_{j}, j=1,2,3$. The eigenspaces $E_{-}^{\mathrm{u}}$ and $E_{+}^{\mathrm{s}}$ intersect trivially since the equation is autonomous. The intersection remains trivial when adding the small perturbations in $\lambda$ and $\nu$. Exploiting exponential convergence of the coefficients of the non-autonomous terms, we may even continue the unstable subspace across the cut $\arg \lambda=\pi$ in a robust way (see $[13,17,14])$. This implies, that for all $\delta$ sufficiently small, we can exclude eigenvalues $\lambda$ in a neighborhood of the origin. This completes the proof of Theorems 1 and 2.

\section{Finite amplitude instability of solitary waves}

The plasma equations (1.1) possess a Hamiltonian structure with Hamiltonian function

$$
H(n, v, \varphi)=\int_{\mathbb{R}}\left(\frac{1}{2} n v^{2}+n \varphi-\frac{1}{2} \varphi_{x}^{2}-\mathrm{e}^{\varphi}\right) \mathrm{d} x
$$


and (formal) degenerate symplectic structure

$$
\omega\left(\left(n_{1}, v_{1}, \varphi_{1}\right),\left(n_{2}, v_{2}, \varphi_{2}\right)\right)=\int_{\mathbb{R}}\left(v_{1} \partial_{x}^{-1} n_{2}+n_{1} \partial_{x}^{-1} v_{2}\right) \mathrm{d} x .
$$

In this symplectic structure, spatial translations are generated by the impulse

$$
J(n, v, \varphi)=\int_{\mathbb{R}} n v .
$$

Both, $J$ and $H$ are conserved quantities for the time evolution of the initial value problem to (1.1).

Solitary waves, as discussed in this article, are critical points of the conserved energy functional

$$
\tilde{H}(n, v, \varphi)=H(n+1, v, \varphi)-c J(n, v, \varphi)
$$

(see Section 2). Unfortunately, the Hamiltonian is strongly indefinite and does not give satisfactory information on global existence or stability of solitary waves. For example, global existence for the initial value problem is not known (local existence in time follows easily from the general results in [18]). However, the Hamiltonian structure is useful in understanding stability at finite amplitude. Heuristically, the family of solitary waves is parameterized by the value of the impulse functional $J$, attained at the solitary wave, or by the speed of the wave $c$. When the function $J(c)$ attains an extremum along the family of the solitary waves, we typically find a saddle-node bifurcation due to an additional eigenvalue crossing the origin, which renders the solitary wave unstable to one side of the extremum. This type of instability has also been observed in water-waves [23,24,31].

This formal reasoning can be extended and made rigorous.

Theorem 3. The solitary wave solutions of the plasma equation (1.1), described in Section 2, are unstable for $c_{\text {crit }}<c<c_{*}$, if $J^{\prime}(c)>0$ for $c<c_{\text {crit }}$ and $J^{\prime}(c)<0$ for $c_{\text {crit }}<c<c_{*}$.

The criterion gives the first instability through a real eigenvalue crossing the imaginary axis. We do not know, if an oscillatory instability renders the solitary wave unstable for $c<c_{\text {crit }}$.

This criterion for instability is frequently used in the literature to prove instability of solitary waves (see [25,7], and the references therein). Similar criteria are known for shock waves in conservation laws [13] and pulse solutions in dissipative systems [28].

Before the proof of Theorem 3, we illustrate the consequences by means of some numerical computations. We computed $J(c)$ numerically from

$$
J(c)=\int_{\mathbb{R}} n_{*} v_{*}=\int_{\mathbb{R}} \frac{\left(c-\sqrt{c^{2}-2 \varphi_{*}(x)}\right)^{2}}{\sqrt{c^{2}-2 \varphi_{*}(x)}} \mathrm{d} x=2 \int_{0}^{\varphi_{\max }(\mathrm{c})} \frac{\left(c-\sqrt{c^{2}-2 \varphi}\right)^{2}}{\sqrt{2\left(c^{2}-2 \varphi\right)\left(\mathrm{e}^{\varphi}+c \sqrt{c^{2}-2 \varphi}-1-c^{2}\right)}} \mathrm{d} \varphi,
$$

where for the substitution of variables in the second equality, we used the identity

$$
\frac{1}{2}\left(\partial_{x} \varphi\right)^{2}-\mathrm{e}^{\varphi}-c \sqrt{c^{2}-2 \varphi}+1+c^{2} \equiv 0,
$$

which holds along the solitary wave. The maximum of the solitary wave $\varphi_{\max }$ is found from

$$
\mathrm{e}^{\varphi_{\max }}+c \sqrt{c^{2}-2 \varphi_{\max }}=1+c^{2}
$$

According to Theorem 3, we found instability for $c_{\text {crit }}<c<c_{\max }$, all wave speeds beyond a critical value $c_{\text {crit }}$. The graph of $J(c)$ is shown in Fig. 3.

We conclude this section with the proof of Theorem 3. 

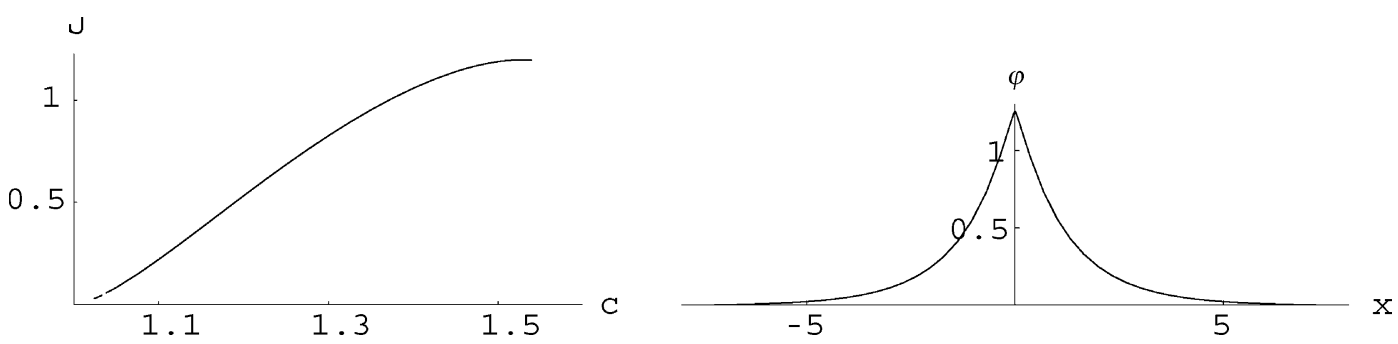

Fig. 3. To the left, the value of the impulse $J$ of the solitary wave as a function of the wave speed $c$ is plotted. Solitary waves are unstable to the right of the maximum. To the right, we show the shape of the $\varphi$-component of the solitary wave at speed $c=c_{\text {crit }}$.

Proof (Theorem 3). We first construct an analytic Evans function $\mathcal{E}(\lambda ; c)$ for the linearization around the solitary wave $\lambda B-L_{*}(c)$, as found in (4.1), for all $\operatorname{Re} \lambda>-\delta$ and $c<c_{\max }$. We then normalize it such that $\mathcal{E}(\lambda ; c) \rightarrow 1$ as $\lambda \rightarrow \infty$. Since $\lambda=0$ is at least algebraically double as an eigenvalue, if the second derivative $\mathcal{E}^{\prime \prime}(0, c)$ of the Evans function is negative for some value of $c$, then $\mathcal{E}(\cdot ; c)$ restricted to the positive real axis is negative for small $\lambda$ and positive for large $\lambda$, which implies existence of an unstable eigenvalue on the positive real axis. For small values of $c$, we conclude from Theorem 2 that $\mathcal{E}^{\prime \prime}(0, c)>0$.

The eigenvalue $\lambda=0$ is at least algebraically double, with kernel given by the derivative of the solitary wave $\partial_{x}\left(n_{*}, v_{*}, \varphi_{*}, \varphi_{*}^{\prime}\right)(\cdot ; c)$ and generalized eigenvector given by the derivative with respect to $c, \partial_{c}\left(n_{*}, v_{*}, \varphi_{*}, \varphi_{*}^{\prime}\right)(\cdot ; c)$. Since for $\lambda=0$, the first two equations can be solved to give $n$ and $v$ as functions of $\varphi$, the kernel can be at most one-dimensional. As a consequence, the eigenvalue $\lambda=0$ is double if and only if the $B \partial_{c}\left(n_{*}, v_{*}, \varphi_{*}, \varphi_{*}^{\prime}\right)^{\mathrm{T}}(\cdot ; c)$ does not lie in the range of $L_{*}(c)$. By Fredholm's alternative, we therefore compute the scalar product with the kernel of the $L^{2}$-adjoint of $L_{*}(c)$, which is given by $\left(-v_{*},-n_{*}-\phi_{*}^{\prime}, \phi_{*}^{\prime}\right)^{\mathrm{T}}$, and find

$$
M=-\int_{\mathbb{R}}\left(v_{*} \partial_{c} n_{*}+n_{*} \partial_{c} v_{*}\right)=-\partial_{c} \int_{\mathbb{R}} n_{*} v_{*}=-J^{\prime}(c) .
$$

Clearly, $M$ vanishes precisely when $\mathcal{E}^{\prime \prime}(0, c)$ vanishes. Following [25,7], one can proceed to show that $M \mathcal{E}^{\prime \prime}(0, c) \neq$ 0 for all values of $c$. This proves the theorem.

\section{Acknowledgements}

The authors gratefully acknowledge financial support by DAAD/Procope, Nr. D/0031082 and F/03132UD. AS wishes to thank the Département de Mathématiques Appliquées, Université Bordeaux 1, for financial support and for providing a fruitful, stimulating atmosphere.

We dedicated this paper to Klaus Kirchgässner. We both are deeply indebted to him for his encouragement and support throughout the years. His enthusiasm has continuously stimulated our work on nonlinear waves, and we express our gratitude by dedicating to Klaus Kirchgässner this joint work on the stability and instability of solitary waves.

\section{References}

[1] J. Alexander, R. Gardner, C.K.R.T. Jones, A topological invariant arising in the stability analysis of traveling waves, J. Reine Angew. Math. 410 (1990) 167-212.

[2] W. Arendt, et al., One-parameter semigroups of positive operators, Lecture Notes in Mathematics, Vol. 1184, Springer, Berlin, 1986.

[3] J.T. Beale, The existence of solitary water waves, Commun. Pure Appl. Math. 30 (1977) 373-389. 
[4] W. Ben Youssef, T. Colin, Rigorous derivation of Korteweg-de Vries-type systems from a general class of nonlinear hyperbolic systems, M2AN Math. Model. Numer. Anal. 34 (2000) 873-911.

[5] T.B. Benjamin, The stability of solitary waves, Proc. Roy. Soc. Lond. A 328 (1972) 153-183.

[6] J.L. Bona, P.E. Souganidis, W.A. Strauss, Stability and instability of solitary waves of Korteweg-de Vries type, Proc. Roy. Soc. Lond. A 411 (1987) 395-412.

[7] T.J. Bridges, G. Derks, The symplectic Evans matrix, and the instability of solitary waves and fronts, Arch. Ration. Mech. Anal. 156 (2001) $1-87$.

[8] V. Coti Zelati, I. Ekeland, E. Séré, A variational approach to homoclinic orbits in Hamiltonian systems, Math. Ann. 288 (1990) 133-160.

[9] W. Craig, An existence theory for water waves and the Boussinesq and Korteweg-de Vries scaling limits, Commun. Partial Diff. Eq. 10 (1985) 787-1003.

[10] J. Evans, Nerve axon equations. iii. Stability of the nerve impulses, Indiana Univ. Math. J. 22 (1972) 577-594.

[11] G. Friesecke, R.L. Pego, Solitary waves on FPU lattices. I. Qualitative properties, renormalization and continuum limit, Nonlinearity 12 (1999) 1601-1627.

[12] C.S. Gardner, G.K. Morikawa, Similarity in the asymptotic behavior of collision-free plasma and water-waves, Technical Report NYO-9082, Courant Inst. Math. Sci., 1960.

[13] R. Gardner, K. Zumbrun, The gap lemma and geometric criteria for instability of viscous shock profiles, Commun. Pure Appl. Math. 51 (1998) 797-855.

[14] M. Haragus, A. Scheel, Finite-wavelength stability of capillary-gravity solitary waves, Commun. Math. Phys. 225 (2002) $487-521$.

[15] E. Infeld, G. Rowlands, Nonlinear waves, Solitons and Chaos, Cambridge University Press, Cambridge, 1990.

[16] T. Kano, T. Nishida, Sur les ondes de surface de l'eau avec une justification mathématique des équations des ondes en eau peu profonde, J. Math. Kyoto Univ. 19 (1979) 335-370.

[17] T. Kapitula, B. Sandstede, Stability of bright solitary-wave solutions to perturbed nonlinear Schrödinger equations, Physica D 124 (1998) 58-103.

[18] T. Kato, Quasi-linear equations of evolution, with applications to partial differential equations, in: Proceedings of the Symposium on Spectral Theory and Differential Equations, Dundee, 1974, Lecture Notes in Mathematics, Vol. 448, Springer, Berlin, 1975, pp. 25-70.

[19] K. Kirchgässner, Wave solutions of reversible systems and applications, J. Diff. Eq. 45 (1982) 113-127.

[20] K. Kirchgässner, Nonlinearly resonant surface waves and homoclinic bifurcation, Adv. Appl. Mech. 26 (1988) 135-181.

[21] E.A. Kuznetsov, A.M. Rubenchik, V.E. Zakharov, Soliton stability in plasmas and hydrodynamics, Phys. Rep. 142 (1986) $104-165$.

[22] Y. Li, D.H. Sattinger, Soliton collisions in the ion acoustic plasma equations, J. Math. Fluid Mech. 1 (1999) 117-130.

[23] M.S. Longuet-Higgins, M. Tanaka, On the crest instabilities of steep surface waves, J. Fluid Mech. 336 (1997) 51-68.

[24] J.W. McLean, Instabilities of finite-amplitude water waves, J. Fluid Mech. 114 (1982) 315-330.

[25] R.L. Pego, M.I. Weinstein, Eigenvalues, and instabilities of solitary waves, Philos. Trans. Roy. Soc. Lond. A 340 (1992) $47-94$.

[26] R.L. Pego, M.I. Weinstein, Asymptotic stability of solitary waves, Commun. Math. Phys. 164 (1994) 305-349.

[27] R.L. Pego, M.I. Weinstein, Convective linear stability of solitary waves for Boussinesq equations, Stud. Appl. Math. 99 (1997) 311-375.

[28] B. Sandstede, Stability of multiple-pulse solutions, Trans. Am. Math. Soc. 350 (1998) 429-472.

[29] C. Sulem, P.-L. Sulem, The nonlinear Schrödinger equation. Self-focusing and wave collapse, Applied Mathematical Sciences, Vol. 139, Springer, New York, 1999.

[30] G. Schneider, E.G. Wayne, The long wave limit for the water wave problem. I. The case of zero surface tension, Commun. Pure Appl. Math. 53 (2000) 1475-1535.

[31] M. Tanaka, The stability of solitary waves, Phys. Fluids 29 (1986) 650-655.

[32] H. Washimi, T. Tanuti, Propagation of ion-acoustic solitary waves of small amplitude, Phys. Rev. Lett. 17 (1966) 996-998. 